\title{
Quasi-Elastic Light-Scattering Investigation of Microemulsions
}

\author{
ERDOGAN GULARI ${ }^{1}$ AND BRIAN BEDWELL
}

Department of Chemical Engineering

AND

\author{
SALIH ALKHAFAJI \\ Department of Nuclear Engineering, University of Michigan, Ann Arbor, Michigan 48109
}

Received October 5, 1979; accepted December 11, 1979

\begin{abstract}
Quasi-elastic light scattering was used to investigate W/O microemulsions of heptane-AOT(bis(2-ethylhexyl)sulfosuccinate sodium salt)-water and $\mathrm{W} / \mathrm{O}$ and $\mathrm{O} / \mathrm{W}$ microemulsions of hexadecane-SDBS (sodium dodecyl benzene sulfonate)- $n$-pentanol-water systems as a function of temperature and dispersed-phase volume. The apparent molecular weights, distribution functions of radii, and diffusion coefficients were determined from the autocorrelation function of the scattered light. In the heptane-AOT-water system it was found that at constant temperature and constant AOT concentration the total interfacial area was independent of the water volume over a fairly large range. The polydispersity and the average hydrodynamic radii increased with temperature and disperse-phase volume for the $\mathrm{W} / \mathrm{O}$ microemulsions. The average droplet radius ranged from 60 to $3000 \AA$. The behavior of the $\mathrm{O} / \mathrm{W}$ microemulsions was found to be more complex. In most of the 11 systems studied the distribution functions were bimodal, indicating the presence of surfactant micelles in equilibrium with microemulsion droplets. The apparent molecular weights determined from intensity measurements and from the average hydrodynamic radii agree well for the heptane-AOT-water systems but the agreement is not good for the other systems.
\end{abstract}

\section{INTRODUCTION AND BRIEF THEORY}

Microemulsions have been studied by a variety of techniques (1-9). In this study we have used the technique of quasi-elastic light-scattering spectroscopy (QLS) $(10,11)$ to study the formation and the polydispersity of the dispersed phase in $\mathrm{O} / \mathrm{W}$ and W/O microemulsions. The systems studied were $\mathrm{W} / \mathrm{O}$ microemulsions of heptaneAOT-(bis(2-ethylhexyl)sulfosuccinate sodium salt)-water and hexadecane-SDBS (sodium dodecyl benzene sulfonate)- $n$ pentanol-water and $\mathrm{O} / \mathrm{W}$ microemulsions of hexadecane-SDBS- $n$ - pentanol-brine $(0.5 \% \mathrm{NaCl})$ systems.

QLS has been used routinely to determine

${ }^{1}$ To whom correspondence and reprint requests should be addressed. the $z$-averaged translational diffusion coefficient and the corresponding hydrodynamic radius of macromolecules, micelles, and colloidal suspensions (11). Unlike the classical light-scattering methods in which the average excess scattered intensity is used to determine the molecular weight and the radius of gyration, QLS looks at the fluctuations in intensity that occur over very short time intervals due to the Brownian motion of molecules or colloidal particles in solution. The time behavior of the fluctuations is described quantitatively by the intensity autocorrelation function $C(\tau)$ :

$$
C(\tau)=\left\langle I^{*}(q, t) I(q, t+\tau)\right\rangle,
$$

where $I$ is the intensity, $q\left(=\left(4 \pi n / \lambda_{0}\right) \sin \theta / 2\right.$; $n$ is the retractive index, $\theta$ is the scattering angle, and $\lambda_{0}$ is the incident wavelength in 
vacuum) is the scattering vector, $t$ is time, and $\tau$ is the delay time.

In the Gaussian approximation the intensity autocorrelation function is related to the field autocorrelation function $g^{1}(\tau)$ by

where

$$
C(\tau)=1+\left|g^{1}(\tau)\right|^{2},
$$

$$
g^{1}(\tau)=\left\langle E_{\mathrm{s}}^{*}(q, t) E_{\mathrm{s}}(q, t+\tau)\right\rangle
$$

and $E_{\mathrm{s}}$ is the scattered electric field. For a suspension of monodisperse spherical particles $g^{1}(\tau)$ is given by

$$
\begin{aligned}
g^{1}(\tau) & =\exp (-\Gamma \tau), \\
\Gamma & =D q^{2},
\end{aligned}
$$

where $D$ is the translational diffusion coefficient of the particles.

Since we always have polydisperse systems Eq. [4] is replaced by

with

$$
g^{1}(\tau)=\int_{0}^{\infty} G(\Gamma) \exp (-\Gamma \tau) d \Gamma
$$

$$
\int_{0}^{\infty} G(\Gamma) d \Gamma=1,
$$

where $G(\Gamma)$ is the normalized distribution function in the $\Gamma$ space. Thus we see that the autocorrelation function contains information about the distribution function of sizes of the dispersed-phase droplets. In theory the most rigorous and the straightforward method of obtaining the distribution function would be the inversion of integral equation [5] to obtain $G(\Gamma)$ from which we can then obtain the size distribution. The inversion, however, is complicated by the infinity limit and the inherent noise; therefore it is not possible. A much used method of analyzing QLS data is the method of cumulants developed by Koppel (12). This method expands the right-hand side of Eq. [3] about the mean, $\bar{\Gamma}$, in a Taylor series:

$$
\begin{aligned}
\ln \left|g^{1}(\tau)\right|=-\tilde{\Gamma} \tau+ & \frac{1}{2 !} \mu_{2} \tau^{2} \\
& -\frac{1}{3 !} \mu_{3} \tau^{3}+\cdots
\end{aligned}
$$

The cumulants $\mu_{2}, \mu_{3}$, etc. are related to the moments of the distribution function. In practice it is hard to go beyond $\mu_{2}$ reliably. Since $\mu_{2}$ is equal to the second moment of the distribution function, $\mu_{2} / \bar{\Gamma}^{2}$ is the variance of the distribution function $G(\Gamma)$ and is a measure of the system's polydispersity. While easy to apply and interpret, the method of cumulants just gives us the variance rather than the distribution function which is the main quantity we are interested in. We have overcome this difficulty by introducing a histogram method of analyzing the intensity autocorrelation function $(13,14)$. The method is general and the only approximation is to replace the continuous distribution curve by a histogram of finite steps:

with

$$
G(\Gamma)=\sum_{j}^{M} a_{j}\left(\Gamma_{j}\right)
$$

$$
\sum_{j}^{M} a_{j}\left(\Gamma_{j}\right) \Delta \Gamma_{j}=1 .
$$

The details of the data analysis to determine the coefficients $a_{j}$ are described elsewhere $(13-15)$.

The $a_{j}$ 's give the relative contribution of each group of particles or droplets with diffusion coefficients between $D_{j}$ and $D_{j}$ $+\Delta D_{j}$, with $D_{j}$ given by Eq. [4a].

For spherical particles or droplets suspended in a solvent the diffusion coefficient is related to the effective hydrodynamic radius $r_{\mathrm{h}}$ of the particle by the StokesEinstein relation:

$$
D=k_{\mathrm{B}} T /\left(6 \pi \eta r_{\mathrm{h}}\right),
$$

where $k_{\mathrm{B}}$ is the Boltzmann constant, $T$ is the absolute temperature, and $\eta$ is the viscosity of the solvent. By introducing the appropriate scattering factors for different spheres we can transform the $G(D)$ to a new number distribution function expressed in terms of the effective hydrodynamic radius $(13-15)$.

\section{EXPERIMENTAL}

The light-scattering spectrometer used in this study was similar to those described in 
the literature (16). The light source was the 514.5-nm line of a Lexel 2-W stabilized argon ion laser. The sample temperature was controlled to $\pm 0.02^{\circ} \mathrm{C}$. The scattered intensity was detected by an ITT FW-130 photomultiplier tube and the correlation functions were measured with a 96-channel single-clipped Malvern correlator. Typical curves took about $10 \mathrm{~min}$ at about $75-\mathrm{mW}$ incident power. The integrated intensities were measured by the same equipment.

The viscosities were measured by a size100 Cannon-Fenske capillary viscometer.

\section{SAMPLE PREPARATION}

The AOT used was Tridom-Fluka purum grade $(99.5 \%)$. The SDBS used was purified from Tridom-Fluka technical-grade SDBS ( $\sim 85 \%$ active) by extraction and crystallization with isopropyl alcohol twice. The estimated purity was about $99 \%$. The heptane, $n$-pentanol, hexadecane, and $\mathrm{NaCl}$ used were all reagent grade and the water was doubly distilled and deionized.

The W/O microemulsions of waterAOT-heptane were prepared by dissolving a fixed amount of AOT in heptane and

\section{TABLE I}

Compositions (wt\%) of the Samples

\begin{tabular}{rrrrr}
\hline $\begin{array}{c}\text { Sample } \\
\text { No. }\end{array}$ & Heptane & AOT & Water \\
\hline 1 & 83.96 & 4.20 & 11.84 \\
2 & 85.25 & & 4.26 & 10.49 \\
3 & 86.80 & & 4.34 & 8.86 \\
4 & 88.50 & & 4.42 & 7.08 \\
5 & 90.10 & & 4.50 & 5.40 \\
& $0.5 \% \mathrm{NaCl}$ & & & \\
& water & SDBS & Hexadecane & $n$-Pentanol \\
& 90.10 & 3.60 & 1.80 & 4.50 \\
6 & 86.81 & 3.47 & 5.21 & 4.51 \\
8 & 82.92 & 3.31 & 8.30 & 5.47 \\
& & & & \\
& Hexadecane & SDBS & Water & $n$-Pentanol \\
& & & & \\
9 & 83.03 & 3.32 & 4.15 & 9.50 \\
10 & 68.97 & 2.76 & 10.34 & 17.93 \\
11 & 67.94 & 2.85 & 10.87 & 18.34 \\
\hline
\end{tabular}

TABLE II

$z$-Averaged Diffusion Coefficients, Hydrodynamic Radii, and Variances for All Samples

\begin{tabular}{clccc}
\hline Sample & $T\left({ }^{\circ} \mathrm{C}\right)$ & $\begin{array}{c}\bar{D} \times 10^{7} \\
\left(\mathrm{~cm}^{2} / \mathrm{sec}\right)\end{array}$ & $\bar{r}_{\mathrm{h}}(\AA)$ & Variance \\
\hline 1 & 15 & 3.44 & 127 & 0.11 \\
& 20 & 3.67 & 143 & 0.13 \\
& 24 & 3.43 & 162 & 0.36 \\
& 27.5 & 2.44 & 243 & 0.81 \\
& 29 & 2.09 & 290 & 1.02 \\
2 & 15 & 3.89 & 112 & 0.11 \\
& 25 & 4.44 & 127 & 0.09 \\
& 35.5 & 2.25 & 294 & 1.16 \\
3 & 25 & 5.60 & 101 & 0.38 \\
& 35 & 4.68 & 140 & 0.59 \\
4 & 15 & 5.09 & 86 & 1.12 \\
5 & 25 & 8.74 & 65 & 0.46 \\
6 & 25 & 0.40 & 615 & 1.25 \\
7 & 25 & 5.45 & 45 & 0.42 \\
8 & 25 & 3.19 & 77 & 0.71 \\
9 & 25 & 0.62 & 122 & 0.04 \\
10 & 25 & 0.46 & 165 & 0.45 \\
11 & 25 & 0.33 & 228 & 0.72 \\
\hline & & & & \\
\hline
\end{tabular}

adding the water afterwards. Sample 1 was prepared by adding the maximum amount of water without forming an emulsion; the other samples 2, 3, 4, and 5 from this system contain progressively less water.

The $\mathrm{W} / \mathrm{O}$ and $\mathrm{O} / \mathrm{W}$ microemulsions of the system water-SDBS- $n$-pentanol-hexadecane were all prepared by mixing weighed quantities of surfactant, water, and oil and titrating to clarity with $n$-pentanol. The water used for the $\mathrm{O} / \mathrm{W}$ microemulsions contained $0.5 \mathrm{wt} \% \mathrm{NaCl}$ as an electrolyte. $\mathrm{NaCl}$ was added to provide screening and eliminate the long-range electrostatic interactions that would otherwise be presene in a surfactant-in-water solution. Table I gives the compositions of all 11 samples used in this study.

\section{RESULTS AND DISCUSSION}

\section{A. Average Diffusion Coefficients and the Hydrodynamic Radii}

Table II gives the diffusion coefficients, the effective hydrodynamic radii, and the 


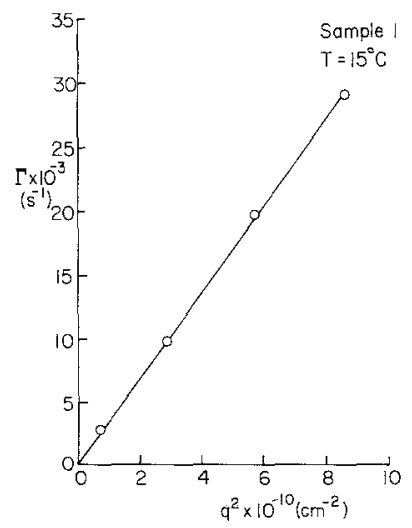

FIG. 1. Plot of the average linewidth $\bar{\Gamma}$ versus the square of the scattering vector $q^{2}$, for sample 1 at $15^{\circ} \mathrm{C}$, showing that the microemulsion droplets are spherical in shape.

variances for all samples obtained from an analysis of the autocorrelation functions by the method of cumulants. While the computation of the diffusion coefficients and the variances from the data is rigorous, computing the effective hydrodynamic radii involves two assumptions. The first is that the dispersed-phase droplets are spherical in shape. This assumption is fairly easy to verify experimentally. For spherical and nearly spherical particles a plot of $\Gamma$, the decay constant of the autocorrelation function, versus $q^{2}$ should be a straight line going through the origin according to Eq. [4a] with a slope equal to the diffusion coefficient. Figure 1 shows the results for sample 1. We see that Eq. [4a] is obeyed perfectly. Similar checks were also made for all samples and they were found to be spherical in shape except for sample 6 which may have nonspherical structures. As a second check of sphericity depolarized scattering intensities were checked and were found to be negligible. The second assumption is about the viscosity to be used in the Stokes-Einstein relationship, Eq. [10]. Equation [10] was originally derived for dilute systems. In concentrated systems $D$ depends on the concentration and as a result will yield the effective hydrodynamic radius if the viscosity of the solvent is used. There is some evidence indicating a fairly weak concentration dependence of $D$ for hard spheres $(17,18)$. Therefore, the $r_{\mathrm{h}}$ computed using Eq. [10] can be taken as a first approximation to the real hydrodynamic radius. Alternatively we can compute the real hydrodynamic radius and $D_{0}$ (the diffusion coefficient at zero concentration) by using one of the several available theoretical relationships between $D$ and $D_{0}(18,19)$ for suspensions of hard spheres. If we use the following expression derived by Altenberger (19)

$$
\begin{aligned}
& D / D_{0}=[1-6 \phi(1-2.2 \phi)] /(1 \\
&\left.-8 \phi+34 \phi^{2}\right),
\end{aligned}
$$

where $\phi$ is the volume fraction of the dispersed phase, for $\phi=0.12$ (typical of all the systems studied) we obtain $D / D_{0} \approx 0.9$. Thus, the $r_{\mathrm{h}}$ calculated by using Eq. [10] alone would be about $10 \%$ larger than the $r_{\mathrm{h}}$ predicted by using Eq. [11] and [10] together. It is difficult to attach any physical significance to the predictions of the hardsphere models because at around $\phi \approx 0.4$ the true hydrodynamic radii predicted by Eq. [11] can be less than the length of the surfactant molecule for some of the other $\mathrm{W} / \mathrm{O}$ microemulsions we are studying at the moment.

There are several important trends in Table II: Sample 1 exists as a single-phase microemulsion up to about $29.2^{\circ} \mathrm{C}$; above that it separates into $\mathrm{W} / \mathrm{O}$ and $\mathrm{O} / \mathrm{W}$ microemulsion phases. We see that as the temperature increases from 15 to $29^{\circ} \mathrm{C}$ the droplet size is more than doubled and we suspect that it gets significantly larger just before phase separation, perhaps behaving like a binary critical system where correlation lengths of a few thousand angstroms have been measured. The increase in size is a consequence of the higher solubility of AOT in heptane at higher temperatures. Thus as the number of surfactant molecules at the $\mathrm{O} / \mathrm{W}$ interface decrease, the diameter of the water core increases in order to keep 
all the water in solution. The fact that the one-phase microemulsion is becoming thermodynamically unstable is also shown by the variances. At the low temperatures we have a small variance characteristic of a thermodynamically stable system and the variance increases 10 -fold as the phaseseparation temperature is approached indicating that no one size is preferred over the others thermodynamically. The diffusion coefficient reaches a maximum at around $20^{\circ} \mathrm{C}$ due to the temperature dependence of the viscosity.

Samples 2, 3, 4, and 5 are essentially the same as sample 1 , the only difference being the decreased water content. If we assume that the equilibrium constant between the interfacial AOT and the AOT dissolved in the continuous phase (heptane) is only a function of temperature, neglecting the surface curvature effects, we arrive at the conclusion that the total surface area should be the same for all of these samples, because the total amount of AOT and heptane is the same. The total interfacial area is given by

$$
A=4 \pi r_{\mathrm{c}}^{2} N=\text { constant }
$$

where $r_{c}$ is the radius of the water core and $N$ is the total number of microemulsion droplets. We can calculate $N$ from a knowledge of the total volume of water and the average core radius of a microemulsion droplet. Using $V_{\mathrm{w}}=4 / 3 \pi r_{\mathrm{c}}^{3} N$ and substituting for $N$ in Eq. [12] we get

$$
\frac{\begin{array}{c}
\text { (volume of } \\
\text { solubilized water })
\end{array}}{r_{\mathrm{c}}}
$$

In our computations we took $r_{\mathrm{c}}=r_{\mathrm{h}}-L_{\mathrm{AOT}}$ (length of an AOT molecule) $\approx r_{\mathrm{h}}-16 \AA$. For $r_{\mathrm{h}}$ the values given in Table II were used. At $25^{\circ} \mathrm{C}$ we obtain $V / r_{\mathrm{c}}$ (relative) $=0.095,0.11,0.12$, and 0.12 respectively for samples $1,2,3$, and 5 (no $r_{\mathrm{h}}$ data for sample 4 at $25^{\circ} \mathrm{C}$ ). At $15^{\circ} \mathrm{C}$ all five samples have $V / r_{\mathrm{c}}=0.12$.

Sample 2 has a phase-separation tempera- ture of about $35.5^{\circ} \mathrm{C}$ and as it is approached the droplet radius increases, paralleling the behavior of sample 1 . We think that there may be a maximum stable droplet size which is reached before phase separation starts for a particular system. The phase-separation temperatures for samples 3, 4, and 5 are above $40^{\circ} \mathrm{C}$ and we did not make extensive temperature studies for these. Samples 4 and 5 are interesting because the refractive indices for these are such that the negative difference between heptane and water is almost compensated for completely by the positive difference between AOT and heptane. Therefore the microemulsion droplets should be transparent if the sizes are uniform. This is confirmed by the very low scattering powers of these samples. Due to the low signals the variances could not be determined reliably.

Samples 6, 7, and 8 are O/W microemulsions and they are the most complicated systems to interpret due to the five components involved. In our analysis we have assumed that the $0.5 \mathrm{wt} \% \mathrm{NaCl}$ provides enough shielding to prevent any long-range electrostatic interactions; as a result Eq. [10] can be used. These samples undergo phase separation when they are cooled, i.e., they have a lower consolute point as opposed to the heptane-AOT-water systems with an upper consolute point. There is no definite trend for these and the average hydrodynamic radius goes through a minimum with sample 7 . Sample 6 was by far the most polydisperse and strongly scattering sample we had, with the dispersedphase volume being about the same as all the other samples. The strong scattering is easily explained by the $615-\AA$ hydrodynamic radius. The very large variances observed are also in agreement with the known long-term instability of these systems.

Samples 9, 10, and 11 are W/O microemulsions and compared to the heptaneAOT-water system we have an additional variable, $n$-pentanol, the cosurfactant. In these systems, the average hydrodynamic 
TABLE III

Molecular Weights

\begin{tabular}{|c|c|c|c|c|c|c|}
\hline \multirow{2}{*}{$\begin{array}{c}\text { Sample } \\
\text { No. }\end{array}$} & \multirow{2}{*}{$\begin{array}{l}\text { Apparent mol wt } \\
\text { from intensity } \\
\text { measurements }\end{array}$} & \multicolumn{3}{|c|}{ Calculated mol wt from $r_{H}$ measurements } & \multirow{2}{*}{$\frac{w t \text { (shell) }}{w t \text { (core) }}$} & \multirow{2}{*}{$\begin{array}{l}\text { wt (shell) } \\
\text { wt (core) } \\
\text { from itput }\end{array}$} \\
\hline & & Shell & Core & Total & & \\
\hline 1 & $9.66 \times 10^{6}$ & $2.16 \times 10^{6}$ & $7.85 \times 10^{6}$ & $10.02 \times 10^{6}$ & 0.276 & 0.354 \\
\hline 2 & $5.40 \times 10^{6}$ & $1.25 \times 10^{6}$ & $3.45 \times 10^{6}$ & $4.70 \times 10^{6}$ & 0.363 & 0.406 \\
\hline 3 & $2.63 \times 10^{6}$ & $7.33 \times 10^{5}$ & $1.55 \times 10^{6}$ & $2.281 \times 10^{6}$ & 0.474 & 0.490 \\
\hline 4 & - & $4.95 \times 10^{5}$ & $8.65 \times 10^{5}$ & $1.36 \times 10^{6}$ & 0.572 & 0.621 \\
\hline 5 & $4.59 \times 10^{5}$ & $2.43 \times 10^{5}$ & $2.97 \times 10^{5}$ & $5.41 \times 10^{5}$ & 0.818 & 0.833 \\
\hline 6 & $2.1 \times 10^{5}$ & - & - & $4.71 \times 10^{8}$ & - & 3.4 \\
\hline 7 & $4.3 \times 10^{5}$ & - & - & $1.82 \times 10^{5}$ & - & 1.09 \\
\hline 8 & $1.4 \times 10^{6}$ & - & - & $9.09 \times 10^{5}$ & - & 0.71 \\
\hline 9 & $1.3 \times 10^{6}$ & $1.64 \times 10^{6}$ & - & $4.16 \times 10^{6}$ & 1.00 & 1.45 \\
\hline 10 & $2.9 \times 10^{7}$ & $3.15 \times 10^{6}$ & - & $1.05 \times 10^{7}$ & 0.43 & 0.52 \\
\hline 11 & $6.3 \times 10^{7}$ & $6.24 \times 10^{6}$ & - & $2.83 \times 10^{7}$ & 0.28 & 0.48 \\
\hline
\end{tabular}

radius and the polydispersity increase with increasing water and alcohol content. These observations are in agreement with those of heptane-AOT-water systems. Sample 9 is the most monodisperse system we have studied.

\section{B. The Apparent Molecular Weights}

Light scattering is probably the most common method of molecular weight determination for macromolecules. For microemulsions, however, the interpretation is not very easy due to the fact that the samples cannot be diluted without changing the dispersed phase. As a result we can only measure an effective molecular weight $M_{\text {eff }}$ given by (20)

$$
\begin{aligned}
\frac{K_{C}}{R_{\mathrm{vv}}}=\frac{1}{M_{\text {eff }}} & =\frac{1}{M_{\text {actual }}}\left(1+q^{2} \frac{\left\langle R^{2} g\right\rangle}{3}\right. \\
& \left.+A_{2} C+A_{3} C^{2}+\cdots\right)
\end{aligned}
$$

where $C$ is the concentration of the dispersed phase, $R_{\mathrm{vV}}$ is the Rayleigh ratio, and $K$ is $4 \pi^{2} n_{0}^{2}(\partial n / \partial C)^{2} /\left(N \lambda_{0}^{4}\right)$, where $N$ is Avogadro's number.

$M_{\text {eff }}$ values computed using Eq. [14] are given in the second column of Table III. Samples 4 and 9 have unreliable $(\Delta n / \Delta C)$ values to compute an effective molecular weight. One can also compute the effective molecular weight from the $r_{\mathrm{h}}$ values given in Table II. We have assumed that the microemulsion droplets consist of an outer shell whose thickness is equal to the length of the surfactant chain and an inner core of water or oil with $r_{\text {core }}=r_{\mathrm{H}}-L_{\text {surfactant }}$. For the shell the density used was a volumeaveraged density of alcohol and surfactant. For samples containing AOT we used a head group area of $55 \AA^{2} /$ molecule and computed the shell weight accordingly. The weights calculated in this fashion are shown in the fifth column in Table III. We see that for samples $1,2,3$, and 5 the agreement between the two methods is very good, indicating that even at volume fractions $\approx 0.1$ these microemulsions behave like ideal solutions.

For $\mathrm{O} / \mathrm{W}$ microemulsions 6,7 , and 8 it is very difficult to draw any conclusions from the results. For 6 , the molecular weight computed from $r_{\mathrm{h}}$ is two orders of magnitude larger. Yet for 7 , the reverse is true; the molecular weight from intensity measurements is almost 10 times larger. For sample 8 , the molecular weight calculated from $r_{\mathrm{h}}$ is again a factor of two and a half orders of magnitude larger. We suspect that electrostatic interactions are still present in these 
systems despite the $0.5 \% \mathrm{NaCl}$ and Eq. [10] cannot be used to get valid $r_{\mathrm{h}}$ values.

$\mathrm{O} / \mathrm{W}$ microemulsion systems 9,10 , and 11 behave as expected. The effective molecular weight increases with increased water content and the molecular weight values calculated from $r_{\mathrm{h}}$ are in reasonable agreement with the intensity results. The discrepancy is probably due to the strong concentration dependence of the scattered intensity and the uncertainties in $C$ and $\Delta n / \Delta C$ and using an average $r_{\mathrm{h}}$.

The shell-weight-to-core-weight ratios were also computed and are shown in Table III along with the theoretical ratios computed from assuming that all the surfactant was at the interface and from measured $n_{\text {alcohol }} /$ $n_{\text {surfactant }}$ ratios. For sample 1, the computed ratio is about $85 \%$ of the theoretical maximum at $24^{\circ} \mathrm{C}$ and increases to about $95 \%$ at $15^{\circ} \mathrm{C}$. For samples 2,3 , and 5 , the computed values are above $90 \%$ of the theoretical maxima, showing that despite the high solubility of AOT in water and in heptane, almost all of it is solubilized at the interface region. The large polydispersity of sample 1 is probably the cause of the low computed ratio.

For W/O microemulsions 9-11, the calculated ratios are somewhat lower than the theoretical maxima, perhaps due to polydispersity.

\section{The Shear Viscosities}

Along with our light-scattering measurements we also made shear viscosity measurements with a Cannon-Fenske viscometer. Table IV shows our results. The viscosities are in good agreement with our light-scattering measurements. In heptaneAOT-water systems the viscosities increase with increasing hydrodynamic radius, with the average viscosity being two and a half times the viscosity of heptane. For $\mathrm{O} / \mathrm{W}$ systems $6-8$, the behavior of $r_{h}$ is precisely paralleled. Sample 6 has by far the highest viscosity, and the viscosity of sample 7 is
TABLE IV

\begin{tabular}{ccc} 
Viscosities & \\
\hline $\begin{array}{c}\text { Sample } \\
\text { No. }\end{array}$ & $\begin{array}{c}\text { Sample viscosity } \\
(\mathrm{cP})\end{array}$ & $\begin{array}{c}\text { Temperature } \\
\left({ }^{\circ} \mathrm{C}\right)\end{array}$ \\
\hline 1 & 1.20 & 21 \\
2 & 1.14 & 21 \\
3 & 1.12 & 21 \\
4 & 1.09 & 21 \\
5 & 1.07 & 21 \\
6 & 8.79 & 25 \\
7 & 2.34 & 25 \\
8 & 5.34 & 25 \\
9 & 4.61 & 25 \\
10 & 4.68 & 25 \\
11 & 5.06 & 25 \\
\hline
\end{tabular}

less than the viscosity of sample 8 . W/O microemulsions $9-11$ behave as expected: viscosity increasing with the dispersedphase volume fraction or with $r_{\mathrm{h}}$.

All our attempts at trying to model the system as a suspension of hard spheres and computing the overall viscosity using the several theoretical expressions given in the literature (21) failed. This was perhaps due to the high degree of polydispersity exhibited by our systems. Only the viscosity of sample 9 , the most monodisperse sample, could be predicted with a high degree of accuracy.

\section{Distribution Functions of Sizes and the Diffusion Coefficients}

One of our main aims in this study was to investigate the polydispersity of the microemulsions quantitatively. Up to now the polydispersity question was left unanswered with previous workers assuming monodispersity. We showed that quasi-elastic light scattering with the histogram method of data analysis could be used to determine quantitative distribution curves in our previous studies $(13-15)$. In this study we have applied the same technique to microemulsions. By solving the integral equation [5] we obtain the distribution function $G(\Gamma)$ in the $\Gamma\left(=D q^{2}\right)$, or diffusion coef- 


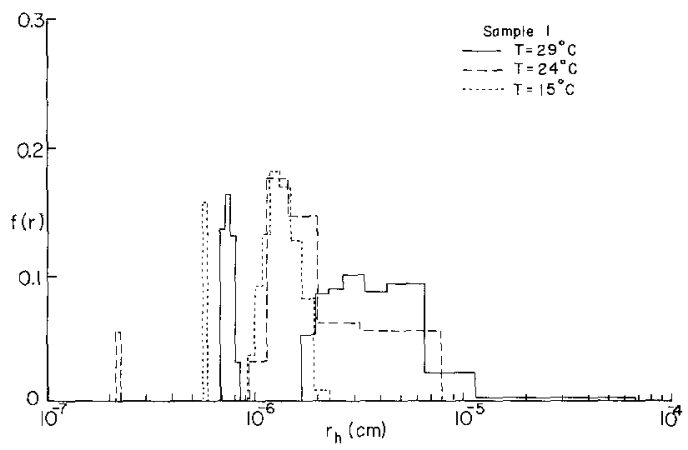

FIG. 2. Distribution functions of size for sample 1 as a function of temperature. The vertical axis $f(r)$ is the relative weight fraction of all the microemulsion droplets with hydrodynamic radii $\left(\mathrm{r}_{\mathrm{h}}\right)_{i}$ to $\left(r_{\mathrm{h}}\right)_{i+1}$.

ficient, space. From the distribution function obtained in the diffusion coefficient space we can make a suitable transformation to any other space we are interested in, such as molecular weight or radius space. The details of the various transformations are described elsewhere (13-15).

Heptane-AOT-water systems exhibit two trends: (i) for a fixed composition the polydispersity increases with temperature and (ii) at a fixed temperature the polydispersity increases with the water-to-AOT ratio. The first effect is due to an increased solubility of AOT in heptane.

Figure 2 shows how the distribution function of the microemulsion droplets changes with temperature for sample 1 . The droplets are increasing in size significantly with temperature and the distribution is also becoming broader. Particle sizes range over two orders of magnitude from micelles of about $20 \AA$ in radius to microemulsion droplets of about $3000 \AA$ in radius. The most interesting aspect, however, is the fact that all three distribution functions are bimodal. We think that the small peaks represent inverse micelles of AOT in heptane existing in equilibrium with the microemulsion droplets, in agreement with observations made by the Kerr effect (22). It is also possible that these fast decays may be due to internal deformation modes of lärge spheres. This possibility will be investigated in the future. Since the micelles scatter relatively little light compared to the giant microemulsion droplets, their exact size is subject to higher uncertainty than the microemulsions. While it is not very obvious from Fig. 2, due to the logarithmic horizontal scale, the weight fraction contained by the micelles is also increasing by almost two orders of magnitude in going from 15 to $29^{\circ} \mathrm{C}$. Figure 3 shows the same phenomena in the diffusion coefficient space which is the primary space.

Figure 4 shows the distribution function for samples 1, 2, and 3 at the same temperature, demonstrating the effect of increased water content on the distribution function. Only the distribution function of sample 1 has a clear indication of the presence of micelles with $r_{\mathrm{h}} \approx 20 \AA$. Sample 2 , which is the most monodisperse one, does not seem to have any micelles. This is perhaps due to their low numbers rather than a complete absence. Sample 3 is not as monodisperse as sample 2 and may have significant amounts of small microemulsion droplets or large micelles with $r_{\mathrm{h}} \approx 55 \AA$.

For the $\mathrm{O} / \mathrm{W}$ systems we did not try to get distribution functions in the $r$ or the molecular weight space due to the unresolved question of electrostatic interactions. The results are shown in the diffusion coefficient space which is the primary data and does not get affected by charge inter-

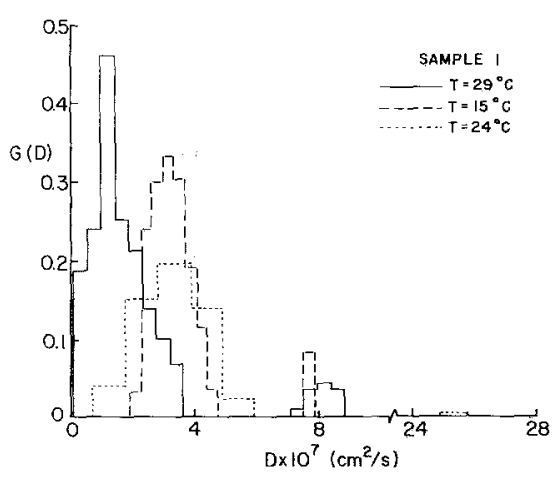

FIG. 3. Distribution functions of the diffusion coefficient for sample 1 as a function of temperature. 
actions. Sample 6 has the most complicated shape, a trimodal distribution function (Fig. 5). Our tentative interpretation is that there are three types of diffusive motion in this system. The first is SDBS micelles or microemulsions undergoing Brownian motion, giving rise to the peak at $1.1 \times 10^{-7} \mathrm{~cm}^{2} / \mathrm{sec}$ (with $r_{\text {eff }} \approx 200 \AA$ ). The second peak is due to the microemulsions diffusing with $D$ $\sim 2.5 \times 10^{-8} \mathrm{~cm}^{2} / \mathrm{sec}\left(r_{\text {eff }} \approx 900 \AA\right)$. The third peak at around $10^{-9} \mathrm{~cm}^{2} / \mathrm{sec}$ ( $r_{\text {eff }}$ $\approx 20,000 \AA$ ) is probably due to cooperative motion of groups of microemulsions and micelles under the influence of long-range electrostatic interactions. Some additional support for this interpretation was obtained when we performed intensity measurements as a function of angle. As we went to lower scattering angles the effective radius of gyration increased to about $2000 \AA$ at $\theta$ $=30^{\circ}$. Due to instrumental limitations we could not reach the limiting value.

$\mathrm{O} / \mathrm{W}$ system 7 is bimodal, showing the presence of micelles with $\bar{D} \approx 8 \times 10^{-7}$ $\mathrm{cm}^{2} / \mathrm{sec}\left(r_{\mathrm{eff}} \approx 29 \AA\right)$ and microemulsions with $\bar{D} \approx 2 \times 10^{-7} \mathrm{~cm}^{2} / \mathrm{sec}\left(r_{\text {eff }} \approx 120 \AA\right)$. The third $\mathrm{O} / \mathrm{W}$ system (sample 8 ) shows the smaller micelles $\bar{D} \approx 10^{-6} \mathrm{~cm}^{2} / \mathrm{sec}\left(r_{\text {eff }}\right.$ $\approx 23 \AA$ ) and slightly larger microemulsion droplets $\bar{D} \approx 1.8 \times 10^{-7} \mathrm{~cm}^{2} / \mathrm{sec}\left(r_{\text {eff }} \approx 130\right.$ $\AA)$. But the significant difference between

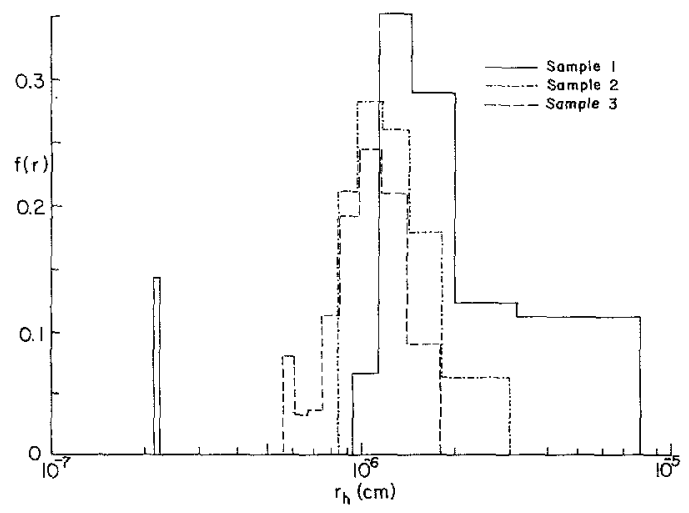

FIG. 4. Distribution functions of size of the dispersed phase for samples 1,2 , and 3 at $25^{\circ} \mathrm{C}$ showing the increase in the average size and polydispersity with water fraction.

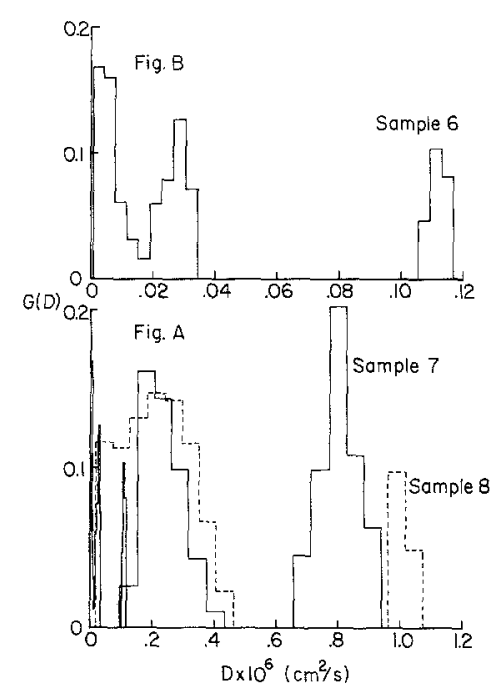

FIG. 5. Distribution functions of the diffusion coefficients for $\mathrm{O} / \mathrm{W}$ systems 6,7 , and 8 . Sample 6 is shown in more detail in the expanded scale of $B$.

samples 7 and 8 is that in sample 7 there are a lot of large micelles, whereas in 8 , due to the higher oil content, most of the surfactant is at the $\mathrm{O} / \mathrm{W}$ interface, leaving very little in water to form micelles. The change in the size of the micelles is also in the right direction. As the concentration of the surfactant is decreased the stable micelle size becomes smaller reaching $\sim 25 \AA$, which should be the radius of a spherical SDBS micelle in water.

The distribution functions of the $\mathrm{W} / \mathrm{O}$ systems with a cosurfactant, systems 9-11, are shown in Fig. 6. Sample 9 has the most monodisperse distribution function of all the systems studied. The range of droplet sizes only changes by a factor of two, and there is no indication of surfactant micelles in solution. Sample 10 is essentially the same except the average size is larger by $40 \%$ and the spread is increased. Sample 11 has the only bimodal distribution in this group, exhibiting the presence of some inverse micelles of size $r_{\text {eff }} \approx 19 \AA$.

\section{v. CONCLUSIONS}

We have investigated three different types of microemulsion systems by a rela- 


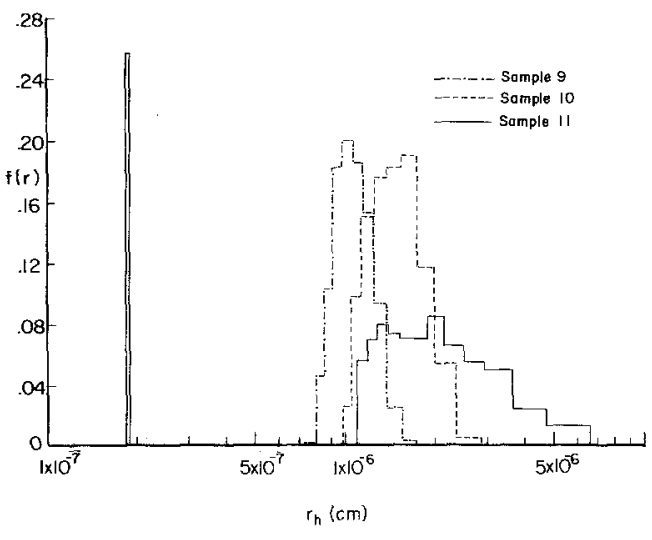

FIG. 6. Distribution functions of size for the $\mathrm{O} / \mathrm{W}$ microemulsions 9,10 , and 11 , showing the increase in the average size and polydispersity with water content. The vertical axis $f(r)$ is the relative weight fraction of droplets with radii $\left(r_{\mathrm{h}}\right)_{i}$ to $\left(r_{\mathrm{h}}\right)_{i+1}$.

tively new and promising tool, QLS. Our important findings are: (i) Depending upon the temperature and composition, the microemulsion systems can have widely varying degrees of polydispersity. (ii) The polydispersity increases with increasing dispersed-phase content and also with decreasing temperature distance from the phase-separation temperature. (iii) The average sizes also increase with the increasing dispersed-phase volume and decreasing temperature distance from the phase-separation temperature. With respect to temperature there are indications that the microemulsions behave somewhat similar to critical systems. (iv) Microemulsions and surfactant micelles or inverse micelles exist in equilibrium with each other. The temperature and the composition determine how much of the surfactant is in the micellar form or at the interface. This equilibrium gives rise to bimodal size distributions. (v) In heptane-AOT-water systems the total surface area is only a function of the surfactant concentration at a fixed temperature. (vi) Perhaps due to the polydisperse nature of the systems studied, the viscosity equations for a suspension of hard spheres could not predict the observed shear vis- cosities except for the case of the most monodisperse system.

We have also shown that QLS is a very powerful method of investigating microemulsions in a nondisturbing fashion, when other methods such as electron microscopy might fail.

Finally we want to point out that the histogram method of analysis gives quantitative information about the distribution of sizes which could not be obtained by the methods of cumulants or just by looking at the scattered intensity.

This work has just shown the bare minimum in applying QLS to microemulsions and we hope to pursue some of the interesting leads in the future.

\section{ACKNOWLEDGMENTS}

We wish to thank Ms. Lori Golze for making the viscosity measurements. Financial support of this research by the National Science Foundation (Grant Eng. 7910267), by an unrestricted grant from the DuPont Corporation, and by a Faculty research grant from the Horace H. Rackham Graduate School is gratefully acknowledged.

\section{REFERENCES}

1. Schulman, J. H., Stoechenius, W., and Prince, L. M., J. Phys. Chem. 63, 1677 (1959).

2. Schulman, J. H., and Riley, D. P., J. Colloid Interface Sci. 3, 383 (1948).

3. Eicke, H. F., and Arnold, V., J. Colloid Interface Sci. 46, 101 (1974).

4. Ekwall, P., Mandell, L., and Fontell, K. F., J. Colloid Interface Sci. 33, 215 (1970).

5. Adamson, A. W., J. Colloid Interface Sci. 29, 261 (1969).

6. Gerbacia, W., and Rosano, H. L., J. Colloid Interface Sci. 44, 242 (1973).

7. Clausse, M., Sheppard, R. J., Boneit, C., and Essex, C. G., in "Colloid and Interface Science" (M. Kerker, Ed.), Vol. II, p. 233. Academic Press, New York, 1976.

8. Sjoblom, E., and Friberg, S., J. Colloid Interface Sci. 67, No. 1 (1978).

9. Shah, D. O., and Hamlin, R. M., Science 3970 , 483 (1971); Shah, D. O., Tamjeedi, L. W., Falco, J. W., and Walker, R. D., AIChE J. 18, 1116 (1972).

10. Cummins, H. Z., and Pike, E. R., Eds., in "Photon Correlation Spectroscopy and Velocimetry." Plenum, New York, 1976: 
11. Chu, B., "Laser Light Scattering." Academic Press, New York, 1974.

12. Koppel, D. E., J. Chem. Phys. 57, 4814 (1972).

13. Gulari, E., Gulari, E., Tsunashima, Y., and Chu, B., J. Chem. Phys. 70, 2965 (1979).

14. Chu, B., Gulari, E., and Gulari, E., Phys. Scr. 19, 476 (1979).

15. Gulari, E., Gulari, E., Tsunashima, Y., and Chu, B., Polymer 20, 347 (1979).

16. Chen, F. C., Yeh, A., and Chu, B., J. Chem. Phys. 66, 1290 (1977).
17. Phillies, G. D. J., Benedek, G. B., and Mazer, N. A., J. Chem. Phys. 65, 1883 (1976).

18. Anderson, J. L., and Reed, C. C., J. Chem. Phys. 64, 3240 (1976).

19. Altenberger, A. R., Chemical Physics 15, 269 (1976).

20. Huglin, M. B., "Light Scattering from Polymer Solutions." Academic Press, New York, 1972.

21. "Colloid Science," Vol. 2. Specialist Periodical Reports, Chem. Soc. Publications, Burlington House, London, 1975.

22. Private communication from H. F. Eicke. 